\title{
Keystone Symposium, Neurodegenerative Diseases: New Molecular Mechanisms 17-22 February 2009
}

\author{
Tom Fagan \\ Alzheimer Research Forum
}

\section{DEATH RECEPTOR LIGAND - NEW ROLE FOR A $\beta$ PP, NEW MODEL FOR AD?}

On Wednesday of the meeting, debate over the last plenary talk continued around the dinner table and well into the poster session. Marc Tessier-Lavigne, Genentech Inc., San Francisco, California, described a novel role for amyloid- $\beta$ protein precursor $(\mathrm{A} \beta \mathrm{PP})$ in neurodegeneration, a role that appears completely independent of amyloid- $\beta(\mathrm{A} \beta)$ toxicity and that might finally explain why only certain neurons bear the brunt of Alzheimer's disease (AD) pathology. Tessier-Lavigne reported that the extracellular domain of $\mathrm{A} \beta \mathrm{PP}$ serves as a ligand for death receptor 6 (DR6), an orphan member of the tumor necrosis factor receptor superfamily. He showed that when the N-terminus of A $\beta$ PP $(\mathrm{N}-$ $\mathrm{A} \beta \mathrm{PP})$ binds DR6, it sets off an apoptotic cascade in embryonic spinal neurons that targets both axons and cell bodies. The work indicates that $\mathrm{N}-\mathrm{A} \beta \mathrm{PP}$ has a role in axonal pruning and neural cell death in development, but it also raises the possibility that similar events occur in mature neurons in the brain. "Finding that an $\mathrm{N}$-terminal fragment of $\mathrm{A} \beta \mathrm{PP}$ is a ligand for DR6 came as a complete surprise, and finding that $\mathrm{A} \beta \mathrm{PP}$ is involved in a self-destruction mechanism like this immediately suggested that perhaps it could contribute to Alzheimer's disease," said Tessier-Lavigne in an interview with this reporter after the talk. The study was coincidentally published in Wednesday's Nature. "This is very intriguing. I think the story is very convincing because there is a lot of very complementary data, biochemical, cell biological, etc., and I do not doubt that it is relevant for A $\beta$ PP biology," said Bart De Strooper, University of Leuven, Belgium, who was at the meeting. He was not involved in the study. "Of course, when you have this type of data I think it is fair to ask how relevant it is for Alzheimer's disease. I think it is too early to say if it is equivalent to the amyloid hypothesis, for example, because there is no human or clinical data," he cautioned.

Tessier-Lavigne and colleagues identified the $\mathrm{N}$ A $\beta$ PP/DR6 interaction when studying neuronal development. The embryo is a tough environment for new neurons with many more being formed than typically needed. Those that do not make proper connections are eventually weeded out by a process that involves axonal pruning and cell death. First author Anatoly Nikolaev and colleagues found that DR6 plays a key role in this process, kicking off an apoptotic pathway in commissural, motor, and sensory neurons that depends on activation of caspase 6 in axons and caspase 3 in the soma only [1]. Nikolaev and colleagues mimicked this axon degeneration by withdrawing trophic support (such as nerve growth factor, or NGF) from neurons in culture, but if they blocked DR6 at the same time - with an antibody, by knocking it down with RNAi, or by genetic knockout - they were able to prevent axonopathy and cell death. Collaborating with Todd McLaughlin and Dennis O'Leary at the Salk Institute, they also showed that DR6 regulates neuron death and axonal pruning not just in cell culture, but also in vivo in a mouse model. 
A $\beta$ PP entered the picture when Tessier-Lavigne and colleagues considered what might activate DR6. The protein is a cell surface receptor with no known ligands. In fact, the researchers were not even sure if a ligand was necessary for its activation, but when they incubated neurons with a soluble DR6 ectodomain construct, it prevented degeneration following trophic factor withdrawal. That suggested that a ligand, mopped up by the soluble DR6 protein, was necessary for the process. In support of this, the researchers found that the DR6-AP - the DR6 ectodomain bound to alkaline phosphatase to act as a reporter - detected proteins $(100 \mathrm{kDa}$ and $35 \mathrm{kDa})$ in conditioned medium following NGF withdrawal suggesting that those proteins may be the DR6 ligands that promote axon degeneration.

The researchers took a leap of faith when they decided to immediately focus on $\mathrm{A} \beta \mathrm{PP}$ as a potential ligand. Tessier-Lavigne explained that in many respects $\mathrm{A} \beta \mathrm{PP}$ fit the bill, as it is shed from the cell surface, it is tied to neurodegeneration already through $\mathrm{AD}$, and it is highly expressed in developing neurons. In initial experiments, the researchers found that DR6-AP bound to $\mathrm{A} \beta \mathrm{PP}$ on the surface of COS- 1 cells. A polyclonal antibody to the $\mathrm{N}$-terminal of $\mathrm{A} \beta \mathrm{PP}$ also recognized the same 100 and $35 \mathrm{kDa}$ proteins that turn up in conditioned medium after trophic deprivation. An antibody to the C-terminal of $\mathrm{sA} \beta \mathrm{PP} \beta$, which is released upon BACE cleavage, also detected the $100 \mathrm{kDa}$ protein in the conditioned medium, and also a $55 \mathrm{kDa}$ protein. The antibody binding patterns suggest that after trophic withdrawal, A $\beta \mathrm{PP}$ is cleaved by BACE to yield the $\sim 100 \mathrm{kDa} \operatorname{sA} \beta \mathrm{PP} \beta$, which is further cleaved, by an unknown protease, to yield the 55 and $35 \mathrm{kDa}$ fragments. Tessier-Lavigne said that it is not clear whether this second cleavage is necessary for activation of the DR6 pathway.

A range of additional experiments supports the idea that $\mathrm{A} \beta \mathrm{PP}$ sets axons off on a suicidal slippery slope. Degeneration of sensory neurons by trophic withdrawal is blocked by antibodies to N-A $\beta$ PP and by knocking down $\mathrm{A} \beta \mathrm{PP}$ by RNAi. BACE inhibitors also blocked the degeneration in cultured neurons, but it could be restored by adding a purified $\mathrm{N}$-terminal fragment of A $\beta$ PP (amino acids 1-286). Finally, the affinity of the $\mathrm{N}$-terminus of $\mathrm{A} \beta \mathrm{PP}$ for DR6 is very high (EC50 for binding is around $4.5 \mathrm{nM}$ ) and the interaction seems fairly specific since the researchers found that $\mathrm{N}-\mathrm{A} \beta \mathrm{PP}$ only reacts with one of the nine other members of the TNF receptor superfamily they tested, p75, and at 60fold lower affinity.

Do these data suggest an entirely new model for neurodegeneration in AD? "What it does is it opens up ad- ditional possibilities for how $\mathrm{A} \beta \mathrm{PP}$ could be involved in the pathological process and therefore opens up new potential therapeutic targets as well," said Tessier-Lavigne in a post-talk interview. The data could, for example, explain why specific neurons are targeted in the disease despite widespread expression of $\mathrm{A} \beta \mathrm{PP}$ in the central nervous system. Tessier-Lavigne showed that DR6 expression in the mature brain is enriched in sites of known AD pathology, including the hippocampus and forebrain cholinergic neurons, suggesting that under certain conditions, shedding of the $\mathrm{A} \beta \mathrm{PP}$ ectodomain might trigger a self-destruct pathway that contributes to neurodegeneration in those very same neurons.

There are other facets of the disease that this model might have more difficulty explaining, however. David Holtzman, Washington University, St. Louis, Missouri, noted during questions that there are familial AD mutations that occur in the $\mathrm{A} \beta$ region of $\mathrm{A} \beta \mathrm{PP}$ and that it would be hard to reconcile how they could fit into this model. Tessier-Lavigne agreed that he needs to determine whether and how such mutations might tie in to his mechanism. He also pointed out that the model is not mutually exclusive of the amyloid hypothesis, and could even be complementary. His laboratory is in the process of crossing DR6 knockout mice with AD mouse models to see what impact the death receptor pathway has on pathology in those systems.

In a post-talk interview, Chris Link, University of Colorado, Boulder, also raised the issue of tau pathology and the formation of neurofibrillary tangles. "I think in some people's view, including mine, Alzheimer's is ultimately a tauopathy, and it is not clear how this model leads to tau hyperphosphorylation," he said. TessierLavigne suggested that caspase 6 activation, which occurs downstream of N-A $\beta$ PP/DR6 binding, might tie $\mathrm{A} \beta \mathrm{PP}$ to tau pathology. He brought up that work from Andrea LeBlanc's laboratory, for example, shows that caspase 6 and tau fragments generated by the protease are elevated in aging and in mild cognitive impairment [2]. "It is possible that [caspase 6 activation] could lead to tau aggregation because there is good evidence that tau fragments play a role, and phosphorylation could be secondary to fragmentation," said Link. "The issue is that although you see neurofibrillary tangles, and certainly tau hyperphosphorylation in neurons, they still look like neurons. If they really had degenerated then they would be gone."

Also, in a News \& Views written for Nature, Donald Nicholson, Merck Research Laboratories, Rahway, New Jersey, noted that caspase 6 can also cleave $\mathrm{A} \beta \mathrm{PP}$ in almost the same location as $\mathrm{BACE}$, raising 
the possibility that the caspase feeds back to generate more N-A $\beta$ PP, thereby amplifying the apoptotic process or spreading it to other cells. "Such potential secondary effects are hard to ignore, particularly because they might be relevant to Alzheimer's disease," writes Nicholson.

Obviously, there is much more work to do to corroborate these results and to explore the mechanism in greater detail. In addition to mouse model work, Tessier-Lavigne said he wants to look for evidence of activation of the DR6 pathway in the adult human brain and particularly in the AD brain. "Since we have additional players in a biochemical pathway, we would like to know if there are any mutations associated with them that represent risk factors in Alzheimer's disease," he added. In fact, DR6 is located very close to a potential susceptibility locus on chromosome 6. "At the same time we are trying to develop potential therapeutic candidates to interfere with steps in this pathway and to use them in preclinical models. If that looks promising, we would obviously consider moving toward the clinic."

\section{PARTNERS IN CRIME - DO A $\beta$ AND PRION PROTEIN PUMMEL PLASTICITY?}

Marc Tessier-Lavigne wowed the crowd by revealing that a soluble piece of $\mathrm{A} \beta \mathrm{PP}$ is a ligand for a cell surface receptor that sets off an apoptotic cascade. Last Friday, it was Stephen Strittmatter's turn to surprise attendees, when he showed that oligomers of $\mathrm{A} \beta$ can bind cellular prion protein (the non-toxic kind). What is more, in the absence of the prion, $\mathrm{A} \beta$ oligomers no longer suppressed long-term potentiation - one of the bestcharacterized $\mathrm{A} \beta$ toxicities. These findings also appear in yesterday's Nature. In an accompanying Nature News \& Views article, Moustapha Cisse and Lennart Mucke of the Gladstone Institute of Neurological Disease, University of California, San Francisco, write that "... the discovery that PrPc may be a mediator in the development of Alzheimer's disease is fascinating, not least from a therapeutic perspective."

Finding $\mathrm{A} \beta$ oligomers bound to cellular prion (PrPc) was absolutely unexpected, Strittmatter told the Alzheimer Research Forum by phone after the symposium. "We clearly think of Alzheimer's and prion disease as being quite separate pathologically and physically. On the other hand, some similarities exist and we are looking forward to functional studies to look into them," he said.
Strittmatter and colleagues, from Yale University, New Haven, Connecticut, made their discovery when screening for proteins that bind $\mathrm{A} \beta$ oligomers. As Strittmatter outlined in his Keystone presentation, the laboratory had previously shown that the Nogo receptor, known for limiting axon regeneration and repair, binds to the $\mathrm{A} \beta \mathrm{PP}$ and to $\mathrm{A} \beta$, but not necessarily oligomers $[3,4]$. To find proteins that specifically bind to oligomeric species of the peptide, which are now widely believed to be the most toxic $\mathrm{A} \beta$ entities, Juha Lauren and colleagues in the laboratory used biotinlabeled oligomers (prepared as per the ADDL method of Bill Klein's group) [5] to screen COS-7 cells expressing a mouse adult brain cDNA library. Screening 200,000 clones generated two strong hits - both expressing the prion protein. The researchers then tested two related proteins, doppel and SPRN, but neither bound to the $\mathrm{A} \beta$ oligomers. A screen of 352 transmembrane proteins (done individually) also failed to detect strong interactions. This method revealed that the A $\beta$ PP homolog APLP1 and the transmembrane protein 30B (TMEM30B) bound the A $\beta$ oligomers with low affinity.

To test whether this oligomer-prion interaction was real, the researchers took advantage of PrPc knockout mice (Prnp-/-), which seem to have normal synaptic plasticity as judged by LTP measurements. At Keystone, Strittmatter showed that after 15-20 days in vitro, cultured hippocampal neurons from wild-type mice bound $\mathrm{A} \beta$ oligomers. This timing corresponds to the emergence of PrPc expression in MAP2-positive dendrites on the neurons, suggesting $\operatorname{PrP}$ is a major $\mathrm{A} \beta$ binding site in these cells. The $\mathrm{A} \beta$ oligomers and the PrPc colocalized, and while the researchers did detect A $\beta$ oligomers on PrPc-negative neurons, binding was reduced by about 50 percent, again indicating $\mathrm{PrPc}$ as a major site for oligomers binding. That binding is not exclusive, though. "Multiple alternative sites, including APLP1, TMEM30A, TMEM30B, RAGE, and other unidentified proteins may explain $\mathrm{A} \beta_{42}$ binding to Prnp-/- neurons," write the authors in the paper.

What might be the functional significance of this prion-A $\beta$ interaction? To test this, the researchers measured long-term potentiation in the Schaeffer collateral pathway of the hippocampus. In hippocampal slices from normal mice, $\mathrm{A} \beta_{42}$ oligomers reduced LTP significantly, but in slices from PrPc-negative animals the oligomers did not. In addition, wild-type slices were protected from the toxic effects of $A \beta$ oligomers if they were first treated with the PrPc antibody 6D11. "Thus, we conclude that PrPc exerts a receptor action acutely 
to mediate $\mathrm{A} \beta_{42}$-oligomer inhibition of synaptic plasticity in the hippocampal slice," write the authors.

How PrPc mediates LTP suppression is unclear, said Strittmatter. One possibility is that the prion- $\mathrm{A} \beta \mathrm{com}-$ plex directly interacts with glutamate receptors and causes their downregulation. The other is that the complex sets off a signal transduction cascade that culminates in glutamate receptor dysfunction. To address this question, the researchers expressed prion protein and glutamate receptors in Xenopus oocytes and then tested the receptor activity by the voltage clamp. "We saw no effect of $\mathrm{A} \beta$ oligomers on glutamate receptors, with or without PrP, so I do not think it is a very direct interaction. I think instead, $\mathrm{A} \beta$ binding to PrP causes changes in calcium, kinases, and endocytosis, the net result of which is glutamate receptor dysfunction," said Strittmatter.

How the two proteins bind is also unknown. These particular oligomers are made up of around 100 monomers and are about $500 \mathrm{kDa}$ in size. One possibility is that the oligomers interact with PrPc through some intermediary. This is unlikely, however, because the researchers found that oligomers bound directly to a purified, immobilized prion chimera comprising the ectodomain of the prion fused to the Fc tail of immunoglobulin $\mathrm{G}$.

There are, of course, smaller oligomers, such as $\mathrm{A} \beta * 56$ isolated by Karen Ashe's group at the University of Minnesota in Minneapolis, oligomers Dominic Walsh first isolated from conditioned medium of $\mathrm{A} \beta$ PP-expressing $\mathrm{CHO}$ cells when he was at Dennis Selkoe's laboratory at Brigham and Women's Hospital, Boston, or even dimers that Ganesh Shankar, Walsh, Selkoe, and colleagues recently isolated from AD patients. Whether any of those bind to PrPc is not known at this point. Strittmatter said that it would be good to test all types of $\mathrm{A} \beta$ for prion binding. His laboratory will look for the functional effects of PrP in mouse models of $\mathrm{AD}$, by crossing PrP nulls with $\mathrm{A} \beta \mathrm{PP}$ transgenic mice and by treating the same transgenic mice with anti-prion antibodies.

Curiously, the region in PrPc, amino acids 95-110, that interacts with $\mathrm{A} \beta$ oligomers also causes profound neurodegeneration when deleted from the prion protein, said Strittmatter [6,7]. "One conclusion from that is there is a natural function for cellular prion, which when perturbed can lead to neurodegeneration," said Strittmatter. "One way to perturb the natural function of prion protein is with toxic prions; a second way is with oligomeric $\mathrm{A} \beta$. It is, in some sense, a receptor for both of these toxic species."
"Lauren and colleagues' observations [8] create fertile ground for future investigations," write Cisse and Mucke [9]. They note that human tau forms complexes with $\operatorname{PrPc}$, which may link $\mathrm{A} \beta$ toxicity to tau, and they observed that the same $\operatorname{PrPc}$ region that binds $\mathrm{A} \beta$ is also cleaved by $\alpha$-secretase [10], which is essential for non-amyloidogenic processing of $\mathrm{A} \beta \mathrm{PP}$. "So one way to prevent both $\mathrm{A} \beta$ production and the activation of downstream mediators by PrPc might be to increase $\alpha$-secretase activity," they write. Array tomography, recently used to localized $\mathrm{A} \beta$ oligomers to dendritic spines in postmortem tissue samples, might also prove a useful model for measuring $\mathrm{A} \beta$-prion interactions in vivo.

\section{LONGEVITY, INSULIN-LIKE GROWTH FACTOR SIGNALING, AND A $\beta$ TOXICITy}

You can never turn back the clock, but you can protect against the dementia risk of advancing age - at least in roundworms and rodents. That was the take-home message from Andrew Dillin's talk on prion protein being a receptor for $\mathrm{A} \beta$. Dillin, from the Salk Institute for Biological Studies, La Jolla, California, talked about how signaling through Daf2, the worm homolog of the mammalian insulin-like growth factor receptor, not only shortens lifespan in Caenorhabditis elegans, but also exacerbates $\mathrm{A} \beta$ toxicity in roundworms, and in mice.

Dillin and colleagues have reported previously that the Daf2 pathway controls aggregation and disaggregation of $\mathrm{A} \beta$ in worms. This Daf 2 effect disappears if either the transcription factor Daf16 or the chaperone Hsf1 is also knocked out. Delving deeper, the Dillin laboratory discovered that the two knockouts affect $\mathrm{A} \beta$ dynamics quite differently, however. The Daf16 knockout results in fewer aggregates of high molecular weight, while the Hsf1 knockout results in more of them. Dillin concluded that Daf16 drives an aggregation pathway, while Hsf1 drives a disaggregation pathway. Relieving suppression of both pathways by knocking out Daf2, which lies upstream of Daf16 and $\mathrm{Hsf} 1$, offers maximal protection against $\mathrm{A} \beta$ toxicity, he said, presumably by removing the most toxic oligomeric species of $\mathrm{A} \beta$.

Dillin next showed data to test the idea that Daf16 acts as an "aggregase." He is using an in-vitro assay for aggregation into which he plants worm extract. The idea is that extracts from worms with less aggregase activity should contain fewer seeds and therefore ac- 
celerate aggregation poorly. This was borne out in tests of Daf16 knockouts - these animals have fewer aggregates and extracts from them seed aggregation poorly. Daf2 knockouts have more aggregates, however, and their extracts seed potently. To test the disaggregase idea, Dillin's laboratory employs the reverse, i.e., measuring loss of thioflavin $\mathrm{T}$ fluorescence as $\mathrm{A} \beta$ aggregates are demolished. Extracts from wild-type and Daf16 knockout worms did have disaggregase activity, and boiling the extract abolished that, suggesting that the phantom disaggregase may be a protein. Hsf1 knockout animals, however, had much lower disaggregase activity than wild-type, in keeping with the idea that Hsf1 activity somehow promotes disaggregation.

Could the same mechanism that protects worms against $\mathrm{A} \beta$ toxicity be at play in mammals? To test this, Dillin and colleagues crossed an $\mathrm{A} \beta \mathrm{PP} /$ presenilin 1 mouse model (see [11]) with insulin-like growth factor receptor (IGFR) heterozygote (homozygote knockouts are lethal). While the AD/IGFR animals do poorly in a Morris water maze test of spatial learning and memory, AD/IGFR +/- mice escape from the maze about four times faster, suggesting that lowering IGF signaling does indeed protect mice against $\mathrm{A} \beta$ toxicity. To examine this in detail, Dillin and colleagues looked at the brains of the mice. They found that at 12-13 months, the AD/IGFR heterozygotes had more NeuN staining than the AD control mice, indicative of less neuronal damage, as well as less evidence of astrocyte activation. As for plaques, though their numbers were equivalent in both strains, the plaques were much denser in the heterozygotes. Dillin interpreted this to mean that these animals are packing the $\mathrm{A} \beta$ better, or that they have a different plaque morphology.

This then raised the question of whether the packing or morphology of plaques might have anything to do with aggregase/disaggregase activity. To address it, the researchers turned to the seeding assay and showed that extracts from the AD/IGFR $+/-$ mice seeded better than from the AD control mice. "It seems that in these [AD/IGFR +/-] animals, the Daf16 pathway is going full blast and protecting in a better way than in controls," Dillin said. Exactly why or how the A $\beta$ dynamic shifts is unclear; however, Dillin showed that brain extract of the protected (IGFR $+/-$ ) animals contained fewer $\mathrm{A} \beta$ dimers and trimers. That not only supports the idea that aggregation is going full tilt, but given recent findings linking dimers with toxicity in humans, it may also help explain why the IGFR+/- animals show less cognitive impairment.

Whether downregulating IGFR signaling might have the same effects in humans is not clear. Interesting- ly, mutations in human IGFR have been found in the very oldest people, suggesting that signaling through this receptor affects longevity in us, as well. But there are reasons to believe that IGFR signaling is beneficial, too. In fact, because of its neurotrophic effects, insulinlike growth factor 1 (IGF-1) has been tried as a potential treatment for motor neuron disease. Unfortunately, clinical trials have been disappointing. Similarly, Merck's MK-677, which boosts circulating IGF-1 levels, has been tested on AD patients, again with disappointing results. The theory was that because AD patients are insensitive to insulin and IGF-1, have low circulating levels of the latter, and because IGF-1 seems to promote clearance of $\mathrm{A} \beta$ from the brain in rodents [12], MK-677 might offer hope of a treatment.

In a final word on the link between IGF signaling and AD, Dillin hinted that knocking out the pathway early on may not be a prerequisite for protection. In worms, blocking Daf 2 even after $\mathrm{A} \beta$ toxicity has begun stops progression of disease, even if it does not bring the animals back to normal. He plans to try the same approach in mice using conditional IGFR knockouts that can be turned on and off.

\section{PULSE-CHASING AD BIOMARKERS, SNARING $\gamma$-SECRETASE TARGETS}

Two things you can find on any Alzheimerologist's wish list are better drug targets and better diagnostics. The drug targets might yield better medicines, and the diagnostics will help identify people in the prodromal phase of the disease, when said medicines may work best. As David Holtzman, Washington University School of Medicine, St. Louis, Missouri, said in his talk, most of the clinical trials going on for $\mathrm{AD}$ right now are conducted after $\mathrm{A} \beta$ accumulation is close to or has reached its maximum, tau accumulation is already substantial, and many neurons have been lost. "We need to be diagnosing AD much earlier," he said.

Holtzman reviewed some of the current biomarker data and also showed how a new pulse-chase method of monitoring changes in $\mathrm{A} \beta$ levels in cerebrospinal fluid (CSF) can help monitor the efficacy of $\gamma$-secretase inhibitors. In his talk, Bart De Strooper, of the University of Leuven and Flaams Instituut voor Biotechnologie in Leuven, Belgium, revealed some protein partners that might be exploited for $\gamma$-secretase inhibition, particularly of its proteolytic processing of $\mathrm{A} \beta \mathrm{PP}$.

Holtzman's laboratory studies antecedent biomarkers, particularly CSF markers such as $\mathrm{A} \beta$ and tau. 
Though CSF concentrations of these markers overlap considerably between controls and patients, it is now well established that AD patients have reduced CSF $\mathrm{A} \beta_{42}$ and elevated levels of CSF tau and p-tau. "The idea is that $\mathrm{A} \beta_{42}$ is going down in the CSF because aggregates form [in the brain] and shift the equilibrium toward plaques," said Holtzman. To see if this is true, his laboratory has correlated CSF levels with brain amyloid load, judged by binding of the PET agent Pittsburgh compound B (PIB). That work showed that nearly every research participant with a high plaque load also had low CSF $\mathrm{A} \beta_{42}$, and almost everyone with high CSF $\mathrm{A} \beta_{42}$ had no plaque load [13]. But the Washington University researchers also follow a third group of people with low CSF $\mathrm{A} \beta_{42}$ and no or few plaques. Holtzman suggested that these people may only have diffuse plaques, which PIB does not bind well, not fibrillary $\mathrm{A} \beta$. In other words, the CSF $\mathrm{A} \beta$ drops before they become PIB positive.

Holtzman said that biomarker data could be used to follow formation or clearance of aggregates as part of monitoring disease progression and/or treatment. One potential way to do that is with microdialysis. Holtzman's group has developed this technique in mice to measure $\mathrm{A} \beta$ changes in the brain's interstitial fluid (ISF) in response to treatments such as a $\gamma$-secretase inhibition. More recently, the researchers have developed a microdialysis probe for screening drugs in vivo. The probe can deliver compounds as well as measure solutes in the ISF, and Holtzman said the technique lends itself to moderate throughput (about 500 compounds a year). As a control, picrotoxin, which increases synaptic activity, increases ISF $\mathrm{A} \beta$ with this system, in keeping with the idea that synaptic activity promotes release of the peptide.

$\mathrm{A} \beta$ levels can and have been measured in human CSF as well, though there is a small snag. "A $\beta$ levels are quite variable in a normal individual's CSF even during a single 24-hour period," said Holtzman. That variation may be linked to synaptic activity, he suggested. One way around that variability may be to simply look at fractional $\mathrm{A} \beta$ synthesis and clearance, rather than total $\mathrm{A} \beta$, which reflects synthesis, clearance, and stability. To do this, Randy Bateman, working originally with Holtzman, has adopted pulse-chase using C13-labelled leucine. The amino acid is injected, readily gets into the $\mathrm{CNS}$, and can be identified in $\mathrm{A} \beta$ peptides following sampling of the CSF. The researchers have tried this technique in both animal models and in humans.

Bateman, Holtzman, and colleagues have used the pulse-chase method to measure the efficacy of a $\gamma$ - secretase inhibitor in humans. While measures of CSF $\mathrm{A} \beta$ did show an effect of the highest dose of the inhibitor, there was a lot of noise in the data, reflecting normally fluctuating $\mathrm{A} \beta$ levels. In contrast, the pulsechase data showed a drop in newly synthesized $\mathrm{A} \beta$ at every dose of the inhibitor tested. The results are highly significant with only five patients per group, Holtzman said. The drug had no effect on clearance of $\mathrm{A} \beta$, which might be expected for a $\gamma$-secretase inhibitor. Holtzman said the data are currently in press in Annals of Neurology.

$\gamma$-secretase is a major drug target for pharma and some academic laboratories studying AD. De Strooper offered up some novel $\gamma$-secretase-related targets for reducing $A \beta$ load. One is the $G$ protein-coupled receptor GPR3, which shuttles $\gamma$-secretase to the cell surface, where it can contribute to amyloidogenic processing of $\mathrm{A} \beta \mathrm{PP}$. GPR3 makes a good drug candidate because it appears to have little to do with processing of Notch, another $\gamma$-secretase substrate, De Strooper said. That work was recently published. Another $\gamma$ secretase-linked target is Aph1B, a component of the presenilin complex. Aph1 comes in three homologs $\mathrm{A}, \mathrm{B}$, and C, and De Strooper and colleagues have shown that Aph1A mouse knockouts look very much like Notch knockouts, while Aph1B/C knockouts appear almost normal. That finding suggests that Aph1A may confer Notch specificity on $\gamma$-secretase, which raises the question of what the other homologs are doing. Could they confer $\mathrm{A} \beta \mathrm{PP}$ specificity on the protease? That idea is borne out by De Strooper's work on Aph1 B/C homologs. In Keystone, he showed that knocking out Aph1B/C partially rescues an AD phenotype. Offspring of Aph1B/C-negative mice bred with $\mathrm{AD}$ mice performed better in the Morris water maze than the AD controls, and the crosses were also protected against $\mathrm{A} \beta$ plaque deposition. The finding suggests that $\gamma$-secretase with Aph1B is a major contributor to the amyloid deposition in the brain, De Strooper said. In support of this, De Strooper showed data from fluorescent lifetime imaging (FLIM) experiments of presenilin reconstituted in $\gamma$-secretase knockout fibroblasts. FLIM reveals how closely connected the different components of the $\gamma$-secretase complex are, and showed that while presenilin was in an open conformation with Aph1A, it was in a closed conformation with Aph1B. De Strooper concluded that Aph1B contributes to $\mathrm{A} \beta$ processing and might be a good target for a selective $\gamma$-secretase inhibitor. 


\section{TAU, HUNTINGTIN - DO PRION-LIKE PROPERTIES PLAY A ROLE IN DISEASE?}

Could prion-like properties of the microtubule binding protein tau explain the phenotypic diversity and the characteristic progression of different tauopathies, such as $\mathrm{AD}$ and frontotemporal dementia? That provocative idea was raised by Marc Diamond, University of California, San Francisco. He was not alone in addressing the possibility. Fellow Californian Ron Kopito from Stanford University suggested prion-like properties might help explain the toxicity of proteins with polyglutamine expansions, such as huntingtin.

The prion-like property Diamond and Kopito referred to is the ability of misfolded proteins to seed aggregation, a property shared by $\mathrm{A} \beta$ and other amyloidogenic proteins. This is typically studied in a cellautonomous fashion, noted Diamond. But what if the seeds could get from one cell to another? Could they then promote aggregation of protein in otherwise healthy cells, a la prions, and could that explain distinct patterns of progression seen in diverse diseases?

To explore this idea, Diamond and colleagues focused on tau. Fibrillar, toxic tau is typically found inside glia and neurons, as in the neurofibrillary tangles of AD, but extracellular NFTs (sometimes called "ghost" tangles) have been found as well, suggesting that the protein has the means of surviving outside the cell and possibly "infecting" others nearby. (Phosphorylated tau is also found in the CSF, which suggests the protein is not restricted to intracellular space). Tau is also associated with 20 or more different tauopathies, each with their own characteristic pathology and progression, and different disease-associated tau mutations can form distinct protein conformations [14], raising the possibility that like prions, there are different strains, or conformers, of tau with distinct disease-causing characteristics.

To test whether tau has prion-like properties, Diamond and colleagues studied tau fibrils formed from wild-type and mutant (P301L/V337M) protein. He showed that when either aggregates in vitro, it forms fibrils with distinct conformations. "This is not surprising, since the amino acid sequences are different," said Diamond. "But can you drive the wild-type into a different conformation by seeding with the mutant?" The answer appears to be yes. He showed that wildtype protein forms what he called $\mathrm{WT}^{*}$ when seeded with mutant fibrils. WT* has a distinct secondary structure, as seen by Fourier transform infrared and circular dichroism spectroscopies. It also exhibits differ- ent fragility under the atomic force microscope than do wild-type fibrils.

The work, some of which was recently published in the February 6 Journal of Biological Chemistry online [15], indicates that tau can exist in at least two conformations. Prions exist in different conformations that are linked to distinct infectious forms [16]; Diamond's work suggests the same may be true for tau. These findings "provide a plausible mechanism for phenotypic diversity seen in tauopathies," he said, though he hastened to add that the existence of different conformers in patient material needs to be confirmed.

If tau can exist in different conformations, could those conformations be transmitted from cell to cell? Diamond and colleagues tested this using rhodamine dye-tagged tau. He showed that tau does indeed gain entry into various cell types, including primary neuronal cultures, and that it may be taken up in an active manner, since the protein often colocalizes with endocytic markers. But are specific misfolding patterns propagated? This was tested by treating cells expressing yellow fluorescent protein (YFP)-tagged normal tau with fibrils made from a fragment of tau comprising the microtubule binding region (MTBR) and a hemagglutinin (HA) tag. The MTBR fibrils caused aggregation of normal tau, as seen by the appearance of yellow puncta in the cells, and these colocalized with the HA-MTBR fibrils taken up by the cells. To test if this conversion can be propagated from cell to cell, Diamond mixed cells expressing either YFP-labeled tau or red fluorescent protein (RFP)-labeled tau. $\mathrm{He}$ showed that after co-culturing the cells and then separating them, those that only express RFP-labeled tau nonetheless have green puncta - indicative of aggregation - suggesting that YFP-tau has made it across the cellular divide. The experiment suggests that tau can move from one cell to another, raising the possibility that in some cases an abnormal form might move in and potentially alter aggregation of normal tau.

Diamond concluded his talk by reiterating the idea that pathogenic tau, such as hyperphosphorylated or mutated tau, is conformationally diverse, aggregationprone, can corrupt normal tau, and can spread between cells. He suggested that if this mechanism is common for other aggregating proteins, it might explain local propagation of cellular dysfunction between neurons and glia - as in ALS - or network degeneration, where protein from one cell can cross a synapse, for example, and corrupt normal protein in a postsynaptic cell. He also suggested that what makes prions unique is not their ability to propagate, but their inherent stability, 
which makes them much more likely to be infectious agents.

Kopito also drew comparisons between prions and another amyloidogenic protein, polyglutamateexpanded huntingtin. Prions self-assemble, have sequence and strain specificity, and can propagate from cell to cell. Expanded huntingtin shares the first two properties, said Kopito, but it was not clear if it can propagate from cell to cell, he said. To test this, Kopito, together with postdoc Pei-Hsien Ren and colleagues in his laboratory, used a nucleation-based assay using synthetic polyglutamine peptides (KKQnKK). In this assay, cells expressing a cyan fluorescent protein (CFP)tagged HttQ25 peptide (which does not aggregate) were treated with fibrils of a rhodamine-tagged Q44 peptide. The idea was that if Q44 fibrils do not behave as prions, then they would probably be taken up by the cell into lysosomes, where they would be degraded, and not interact with normal cytosolic huntingtin. However, if they could gain access to the cytosol, then they could co-opt the Q25 proteins into their fibrils, effectively spreading protein misfolding. In fact, this is what Kopito and colleagues saw. Within one hour of treating HEK293 cells, the normally diffuse CFP-tagged Htt fragment redistributed into puncta that colocalized precisely with Q44 aggregates taken up from outside. After 24 hours, nearly 100 percent of the cells exhibited this phenotype. The effect is sequence specific, since neither aggregates of $\mathrm{A} \beta$ nor Sup35, the yeast prion, could convert CFP-HttQ25 to a fibrillar form.

So do polyglutamine expanded proteins behave like prions? To test this, Kopito and colleagues looked at another prion property - inheritance. Different prion strains, such as found in the yeast prion Sup35, confer different phenotypes on their host, and these phenotypes can be propagated from generation to generation. Ren and colleagues tested if that might be true in cells infected with polyglutamine expanded huntingtin. They treated CFP-HttQ25-expressing HEK293 cells with KKQ44KK aggregates and then allowed the cells to divide. While initially all the cells had CFP puncta, indicative of induced aggregation of the httQ25 chimera, the number of cells with puncta declined with each round of cell division, consistent with dilution of the original innoculum. But curiously, after about 30 generations, the number of CFP-Htt puncta per cell rose and was significantly higher than if the cells were infected with a control treatment, such as $\mathrm{A} \beta$ aggregates. The experiment suggested that the aggregation phenotype can be passed on to the next generation. Persistent aggregation is a new and inheritable phenotype, suggested Kopito.
So are tau, mutant huntingtin, and perhaps other amyloidogenic proteins really prions? Kopito was hesitant to call polyglutamine expanded proteins prions, preferring the term prion-like. Heather True, Washington University School of Medicine, St. Louis, Missouri, who studies yeast prions and was not involved in either Kopito's or Diamond's work, was at the Keystone symposium. In a post-meeting interview with ARF, True said, "We can think about these proteins as having prion-like mechanisms, but it is hard to use the word 'prion' per se." One of the main differences is localization, she said. "It is an accessibility issue more than anything else. I think that the underlying mechanisms are really very similar, but realistically, with PrP being a cell surface protein, the accessibility and availability of substrate, which really makes for a strong persistent infection, is a huge factor."

\section{TOXIC OR TRUANT - KEEPING TAU ON TRACK}

Is it time to stop focusing on $\mathrm{A} \beta$ monotherapies for $\mathrm{AD}$ and start looking to tau therapies as well? That was the take-home message from Karen Duff's talk. At Keystone, Duff offered up some potential drug targets that might attenuate tau toxicity, keeping the protein from potentially disastrous phosphorylation, which drives tau into NFTs. The importance of keeping tau in its native form was also brought home by Matthew Elrick, University of Michigan, Ann Arbor. He showed how loss of normal tau exacerbates pathology in a mouse model of another tauopathy, the lysosomal storage disorder, Niemann-Pick disease type C. That finding indicates that there may be mechanisms independent of tau aggregation that contribute to pathology in tau disorders.

In addition to $\mathrm{AD}$, tau pathology is associated with over 20 different diseases, or tauopathies, making taubased treatments a potential panacea for many neurological disorders. Niemann-Pick's, which is just one of those diseases, is caused by a mutation in either NPC1 or NPC2, proteins involved in lipid trafficking and cholesterol homeostasis. In humans, NiemannPick's causes progressive neurodegeneration and is associated with NFTs. In NPC1-deficient mouse models of the disease there are no NFTs, but tau kinases are activated and hyperphosphorylation of tau is rampant. Elrick suggested that the mice die before tangles get a chance to form. 
To investigate the role of tau in Niemann-Pick's, Elrick and colleagues crossed NPC1-null mice with tau knockouts. Surprisingly, he showed that the NPC1/tau double null mice have an enhanced phenotype and die significantly earlier than the NPC1 knockout animals, suggesting that tau has a protective role. To figure out what that might be, Elrick looked at autophagy, which is known to be partially elevated in NiemannPick's - LC3II, a marker of the autophagic process is upregulated, as are autophagosomes, but flux through the pathway is reduced by about $75 \%$, indicating that processing through the pathway is not robust.

In the tau-deficient mice, Elrick found that LC3II levels were normal. He also showed that induction and flux through the autophagic pathway is reduced in NPC1/tau-deficient human fibroblasts. Since protein degradation through the autophagy pathway depends on microtubule-associated movement, Elrick concluded that tau hyperphosphorylation negatively impacts autophagy and so contributes to disease pathology.

One way to prevent that tau phosphorylation is to block the kinases that are involved. Duff showed that one potential target, the kinase Cdk5, is, in fact, less than ideal. Cdk5, and its co-activator $\mathrm{p} 25$ have been implicated in AD pathology, and there are conflicting reports that p25 is elevated in AD patient brains, but Duff pointed out that blocking this kinase actually results in more tau phosphorylation because another tau kinase, glycogen synthase kinase $3 \beta$ (GSK $3 \beta$ ), is activated. Blocking GSK3 $\beta$ - with lithium chloride, for example - might be a better approach, suggested Duff [17]. But she cautioned that any tau therapy might have to be given early because once tangles form, they can continue to accumulate even after tau is suppressed. This could be one reason why AD clinical trials are failing, suggested Duff.

One way to deal with those later consequences of tau pathology would be to find compounds that disaggregate tau fibrils. Duff has developed organotypic cell cultures using tau transgenic mice to screen for such compounds. The tissue can be taken from 10-day-old animals and cultured for months, Duff said. Taking tissue from both sides of the brain allows for both control and test samples. With this method, Duff and colleagues have discovered $\mathrm{C} 11$, a cyanine dye derivative that reduces fibrils. Duff showed that at low doses, C11 reduces the length and number of tau filaments formed in cultures, as seen in the electron microscopes following Sarkosyl extraction of the tissue. The low dose does not appear to have any detrimental effect on the cells. High doses of $\mathrm{C} 11$ seem to have the opposite effect, however, promoting tau aggregation. Duff plans to further study $\mathrm{C} 11$ and use the organotypic culture method to find better tau disaggregating compounds.

\section{MORE THAN MERE NUCLEOTIDES - MIRNAS AS MASTER REGULATORS, PART 1}

If a keystone was to be found at the recent symposium, it may well have been microRNAs (miRNAs). These non-coding RNAs have been cropping up almost anywhere researchers care to look, and because they can simultaneously regulate a multitude of seemingly unconnected genes and pathways, scientists are wondering if they may be a common link that spans disparate aspects of complex neurodegenerative diseases. Whether that turns out to be the case remains to be seen, but from control of $\mathrm{A} \beta$ production to protection from excitotoxicity to control of adult neurogenesis, miRNAs were central to many of the Keystone talks at last month's symposium.

Alzforum readers have become familiar with miRNAs in part because of work from Bart De Strooper's laboratory in KU Leuven, Belgium. This group has shown that levels of some 20 miRNAs are low in AD patients and that several of these microRNAs can theoretically bind $\mathrm{A} \beta \mathrm{PP}$, presenilin, or $\beta$-secretase (BACE) genes. At Keystone, De Strooper reviewed some of the evidence linking these miRNAs to BACE, the protease that kicks off amyloidogenic processing of $\mathrm{A} \beta \mathrm{PP}$. MicroRNAs miR 29a and 29b-1 are downregulated in some AD patients, and this correlates with high BACE expression. De Strooper showed that when added to cells in culture, these miRNAs downregulate BACE and $\mathrm{A} \beta$ production, suggesting that they could play important regulatory roles in the pathophysiology of AD.

The human genome contains at least 450 miRNAs, said De Strooper, and they regulate thousands of target genes. He suggested that they act like mini-cellular rheostats, dampening gene expression in response to external stimuli. The role of miRNAs, because they have multiple targets, can be profound. "Loss of miRNAs sets the stage for the "multiple hit' hypothesis," said De Strooper, adding that it will be important to look for other genes that may be regulated by these miRNAs in AD and in other diseases, as well.

The talk by Valina Dawson, Johns Hopkins University, Baltimore, Maryland, was germane to both miRNAs and the multiple hit hypothesis. Dawson has been working on factors that help cells survive a non-lethal, 
stressful stimulus. The multiple hit hypothesis suggests that several such stimuli might combine to deal a lethal blow to cells. By identifying pathways activated during non-lethal "preconditioning," Dawson, who coorganized this Keystone symposium, hopes to identify mechanisms that could be exploited to promote neuronal survival in neurodegenerative conditions, such as Parkinson's disease. She reported that one such pathway involves the transcription factor NFI-A (nuclear factor I/A). NFI/A is upregulated in primary cortical neurons exposed to stressful levels of the glutamate receptor agonist N-methyl-D-aspartate (NMDA). Dawson showed that knocking out the transcription factor weakens the preconditioning protection afforded by non-lethal doses of NMDA, blunting cell survival in the process; conversely, overexpressing NFI/A increases survival.

The NFI/A protective effects appear intimately linked to the protein's role as a transcription factor, since cells expressing NFI/A mutants without the ability to bind DNA derive no protection from the preconditioning. Dawson also showed that the transcription factor is protective in vivo, since NFI/A+ / - heterozygote mice are less resistant to NMDA toxicity and develop bigger lesions in response to the glutamate agonist.

The microRNA connection comes in because NFI/A has a binding site for miR223. Would this miRNA affect survival of neurons challenged with NMDA? In fact, that is just what Dawson showed. Not only does overexpressing miR223 block the preconditioning effect, but preconditioning alone alters miR223 in a biphasic manner and in diametrical opposition to changes in NFI/A expression. The result suggests that the preconditioning stress stimulus is acting through this microRNA to relieve suppression of NFI/A and protect cells. However, the scenario is slightly more complicated. Dawson, in collaboration with Fernando Camargo at the Whitehead Institute, Cambridge, Massachusetts, has generated miR223 knockout animals. They are, in fact, more - not less - sensitive to glutamate toxicity. "The knockout is likely having pleiotropic effects," said Dawson, which would be in keeping with miRNAs having multiple targets. One of miR223's targets is, for example, a death effector protein. Dawson is planning to use microRNA sponges, transcripts that recognize and soak up miRNAs, to mop up miR223 in cells and to tease out its pleiotropic effects.

In other presentations, researchers outlined roles for miRNAs in Parkinson's disease, Huntington's disease, and adult neurogenesis.

\section{MORE THAN MERE NUCLEOTIDES - MIRNAS AS MASTER REGULATORS, PART 2}

MicroRNAs were a common emerging theme. These small non-coding RNAs generally dial down gene expression by silencing messenger RNAs, and there is some hope they may eventually offer new ways of treating a wide variety of disorders, including neurodegenerative diseases such as AD. For example, at Keystone, Bart De Strooper, KU Leuven, Belgium, reviewed evidence from his laboratory linking miRNAs to regulation of BACE activity, and Valina Dawson, Johns Hopkins University, Baltimore, Maryland, showed a role for miRNAs in regulating pathways that protect cells from stress.

One neurodegenerative disease that might benefit from activating pro-survival pathways is Parkinson's disease. It has already been linked to non-coding RNAs. These regulate dopamine receptor levels and developmental pathways that lead to a Parkinson-like phenotype when perturbed. MicroRNA miR133b is reduced in Parkinson's disease [18], while an FGD20 gene mutation found in some Parkinson's disease patients is in a binding site for miR433 [19]. In his short talk, Wim Mandemakers, KU Leuven, Belgium, offered a link between microRNAs and $\alpha$-synuclein, the major component of Lewy body pathology. Since elevated expression of $\alpha$-synuclein by itself is sufficient to cause Parkinson's, and microRNAs generally suppress expression, the notion that microRNAs might be involved in $\alpha$-synuclein regulation is worth exploring, suggested Mandemakers.

Mandemakers used a bioinformatics screen to search for microRNAs that might bind the $\alpha$-synuclein (SNCA) sequence. This identified miR7 and miR153 as possible matches. Using a luciferase gene construct containing the SNCA 3' untranslated region, Mandemakers showed that both microRNAs suppressed luciferase expression in HeLa cells and miR153 also suppressed expression of the reporter in SH-SY5Y neuroblastoma cells, suggesting the microRNA/SNCA interaction may be functionally significant. Then he showed that overexpressing miR153 in either SH-SY5Y or PC12 cells suppressed $\alpha$-synuclein.

Whether miR153 has any influence on Parkinson pathology is as yet unclear. Mandemakers did show that the microRNA is expressed in the substantia nigra, a small region of the brain where Parkinson pathology is rampant, but the microRNA is more robustly expressed in the total brain. Analysis of miR 153 knockdown mice 
is underway to determine how the microRNA affects $\alpha$-synuclein expression in vivo, he said.

Fiona Doetsch, Columbia University, New York, reported how different microRNAs, in different places in the brain, are crucial for adult neurogenesis, a phenomenon that holds promise for treating neurodegenerative conditions in the future. Doetsch showed that at least three microRNAs are involved - 181a, 181b, and 124 - each apparently playing a different role in the neurogenesis process. This process starts in the subventricular zone (SVZ) with astrocytic stem cells, which generate transit amplifying cells that wind their way via the rostral migratory stream (RMS), ending up as neuroblasts in the olfactory bulb. The three miRNAs may have specific roles in each of the three cells, suggested Doetsch: In the subventricular zone the astrocytic neural precursors predominantly express miR181b; the rapidly dividing transit-amplifying cells express miR181a; and the neuroblasts express miR124. It was the role of this miRNA that Doetsch focused on. She showed that it was not simply the site of miR124 expression that was crucial, but also the timing. Some of this work recently appeared in the March 15 Nature Neuroscience online [20].

Doetsch reported that injecting an miR124-expressing retrovirus into the SVZ led to an induction of neurogenesis in mice, while adding antisense miR124 to purified stem cells in culture caused a significant increase in the number of dividing cells but a decrease in the number of neurons - cell survival was unchanged. The results suggested that miR124 promotes differentiation into neurons. To test if miR124 regulates the process or its timing, Doetsch and colleagues again turned to in-vivo experiments. They completely ablated neurogenesis by using miniature osmotic pumps to flood the brain for six days with the anti-mitotic agent Ara-C, a cytosine analog that blocks DNA replication. After this treatment, astrocytic stem cells in the SVZ that were not dividing during the treatment survive and begin to repopulate the RMS and the olfactory bulb with neuroblasts, which begin to appear after four and a half to five days. However, if antisense miR124 is pumped into the brain immediately after Ara-C treatment, then the number of neuroblasts is almost zero five days later, but the number of transit amplifying cells is roughly doubled. This is in keeping with miR124 acting as a pro-differentiation switch. Interestingly, another two days later, there is a huge increase in the number of neuroblasts formed. These results indicate that miR124 controls not the differentiation into neurons, but the timing of that differentiation, said Doetsch.
How does miR124 exert its control? To address this next question, Doetsch studied targets of the microRNA, including the genes Dix-2, Jag-1, Sox-9, and Pea-15. All of these were knocked down in the presence of the microRNA. Sox-9 is of particular interest to Doetsch because it is expressed in astrocytes, and its mRNA, but not the protein, is found in neuroblasts. Using a Sox-9 gene construct lacking the region that binds miR124, Doetsch showed that overexpression of the gene abolished neurogenesis, and adding miR124 did not rescue this effect. The results suggest that the major effect of miR124 on neurogenesis is probably through suppression of Sox-9, Doetsch said.

Whether these findings will lead to future therapies that boost neurogenesis in the adult brain remains to be seen. Other miRNAs, by contrast, have already been studied in this regard. Beverly Davidson, University of Iowa, Iowa City, reported that artificial miRNA strategies may be one way to go in the treatment of Huntington's disease. Davidson noted some of her previous work showing that the short hairpin RNA sh2.4, which targets the second exon of the huntingtin $\mathrm{mR}$ NA, reduces expression of the protein when placed in an miRNA expression system. This artificial miRNA protected striatal neurons against huntingtin toxicity in a mouse model of Huntington's disease [21].

One advantage of miRNAs is that they can target different alleles of the same gene, be they mutants or transgenes. Davidson showed more recent evidence that the sh2.4 artificial miRNA can counteract both human and mouse huntingtin in a transgenic mouse model of the disease established by David Borchelt [22]. Injecting the RNA at seven weeks of age silenced both mRNAs by 20 weeks, and the mice showed a benefit on the rotarod. While control mice progressively deteriorated between 14 and 18 weeks, the treated mice maintained their performance during this time. They also lived longer, said Davidson. Some of this work recently appeared in the February 24 Molecular Therapy online [23].

It is too early to say whether the study of miRNAs will identify additional novel pathways or drug targets that can actually be exploited as treatments for AD or other neurodegenerative diseases. In the meantime, the talks at the Keystone symposium illustrated just how manifold the roles of these small actors are in the brain. Given that this field is still young, that microRNAs are strictly regulatory, and that they have pleiotropic effects, it may well turn out that these micro players have a macro impact on the field. 


\section{REFERENCES}

[1] Nikolaev A, McLaughlin T, O’Leary DD, Tessier-Lavigne M (2009) APP binds DR6 to trigger axon pruning and neuron death via distinct caspases. Nature 457, 981-989.

[2] Albrecht S, Bourdeau M, Bennett D, Mufson EJ, Bhattacharjee M, LeBlanc AC (2007) Activation of caspase-6 in aging and mild cognitive impairment. Am J Pathol 170, 1200-1209.

[3] Park JH, Gimbel DA, GrandPre T, Lee JK, Kim JE, Li W, Lee DH, Strittmatter SM (2006) Alzheimer precursor protein interaction with the Nogo-66 receptor reduces amyloid-beta plaque deposition. J Neurosci 26, 1386-1395.

[4] Park JH, Widi GA, Gimbel DA, Harel NY, Lee DH, Strittmatter SM (2006) Subcutaneous Nogo receptor removes brain amyloid-beta and improves spatial memory in Alzheimer's transgenic mice. J Neurosci 26, 13279-13286.

[5] Chromy BA, Nowak RJ, Lambert MP, Viola KL, Chang L, Velasco PT, Jones BW, Fernandez SJ, Lacor PN, Horowitz P, Finch CE, Krafft GA, Klein WL (2003) Self-assembly of Abeta(1-42) into globular neurotoxins. Biochemistry 42, 12749-12760.

[6] Baumann F, Tolnay M, Brabeck C, Pahnke J, Kloz U, Niemann HH, Heikenwalder M, Rülicke T, Bürkle A, Aguzzi A (2007) Lethal recessive myelin toxicity of prion protein lacking its central domain. EMBO J 26, 538-547.

[7] Li A, Christensen HM, Stewart LR, Roth KA, Chiesa R, Harris DA (2007) Neonatal lethality in transgenic mice expressing prion protein with a deletion of residues 105-125. EMBO J $\mathbf{2 6}$, 548-558.

[8] Lauren J, Gimbel DA, Nygaard HB, Gilbert JW, Strittmatter SM (2009) Cellular prion protein mediates impairment of synaptic plasticity by amyloid-beta oligomers. Nature 457, 1128-1132.

[9] Cisse M, Mucke L (2009) A prion protein connection. Nature 457, 1090-1091.

[10] V incent B, Cisse MA, Sunyach C, Guillot-Sestier MV, Checler F (2008) Regulation of betaAPP and PrPc cleavage by alphasecretase: mechanistic and therapeutic perspectives. Curr Alzheimer Res 5, 202-211.

[11] Borchelt DR, Ratovitski T, van Lare J, Lee MK, Gonzales V, Jenkins NA, Copeland NG, Price DL, Sisodia SS (1997) Accelerated amyloid deposition in the brains of transgenic mice coexpressing mutant presenilin 1 and amyloid precursor proteins. Neuron 19, 939-945.

[12] Carro E, Trejo JL, Gomez-Isla T, LeRoith D, Torres-Aleman I (2002) Serum insulin-like growth factor I regulates brain amyloid-beta levels. Nat Med 8, 1390-1397.

[13] Li G, Sokal I, Quinn JF, Leverenz JB, Brodey M, Schellenberg GD, Kaye JA, Raskind MA, Zhang J, Peskind ER, Montine
TJ (2007) CSF tau/Abeta42 ratio for increased risk of mild cognitive impairment: a follow-up study. Neurology 69, 631639.

[14] von Bergen M, Friedhoff P, Biernat J, Heberle J, Mandelkow EM, Mandelkow E (2000) Assembly of tau protein into Alzheimer paired helical filaments depends on a local sequence motif ((306)VQIVYK(311)) forming beta structure. Proc Natl Acad Sci U S A 97, 5129-5134.

[15] Frost B, Ollesch J, Wille H, Diamond MI (2009) Conformational diversity of wild-type tau fibrils specified by templated conformation change. J Biol Chem 284, 3546-3551.

[16] Tanaka M, Chien P, Naber N, Cooke R, Weissman JS (2004) Conformational variations in an infectious protein determine prion strain differences. Nature 428, 323-328.

[17] Noble W, Planel E, Zehr C, Olm V, Meyerson J, Suleman F, Gaynor K, Wang L, LaFrancois J, Feinstein B, Burns M, Krishnamurthy P, Wen Y, Bhat R, Lewis J, Dickson D, Duff $\mathrm{K}$ (2005) Inhibition of glycogen synthase kinase-3 by lithium correlates with reduced tauopathy and degeneration in vivo. Proc Natl Acad Sci U S A 102, 6990-6995.

[18] Kim J, Inoue K, Ishii J, Vanti WB, Voronov SV, Murchison E, Hannon G, Abeliovich A (2007) A MicroRNA feedback circuit in midbrain dopamine neurons. Science 317, 12201224.

[19] Wang G, van der Walt JM, Mayhew G, Li YJ, Züchner S, Scott WK, Martin ER, Vance JM (2008) Variation in the miRNA433 binding site of FGF20 confers risk for Parkinson disease by overexpression of alpha-synuclein. Am J Hum Genet $\mathbf{8 2}$, 283-289.

[20] Cheng LC, Pastrana E, Tavazoie M, Doetsch F (2009) miR124 regulates adult neurogenesis in the subventricular zone stem cell niche. Nat Neurosci 12, 399-408.

[21] McBride JL, Boudreau RL, Harper SQ, Staber PD, Monteys AM, Martins I, Gilmore BL, Burstein H, Peluso RW, Polisky B, Carter BJ, Davidson BL (2008) Artificial miRNAs mitigate shRNA-mediated toxicity in the brain: implications for the therapeutic development of RNAi. Proc Natl Acad Sci U S A 105, 5868-5873.

[22] Schilling G, Becher MW, Sharp AH, Jinnah HA, Duan K, Kotzuk JA, Slunt HH, Ratovitski T, Cooper JK, Jenkins NA, Copeland NG, Price DL, Ross CA, Borchelt DR (1999) Intranuclear inclusions and neuritic aggregates in transgenic mice expressing a mutant $\mathrm{N}$-terminal fragment of huntingtin. Hum Mol Genet 8, 397-407.

[23] Boudreau RL, McBride JL, Martins I, Shen S, Xing Y, Carter BJ, Davidson BL (2009) Nonallele-specific silencing of mutant and wild-type Huntingtin demonstrates therapeutic efficacy in Huntington's disease mice. Mol Ther 17, 1053-1063. 